\title{
Augmented Reality in Surgical Procedures
}

\author{
E. Samset ${ }^{\mathrm{a}}$, D. Schmalstieg ${ }^{\mathrm{b}}$, J. Vander Sloten ${ }^{\mathrm{c}}$, A. Freudenthal ${ }^{\mathrm{d}}$, \\ J. Declerck ${ }^{\mathrm{e}}$, S. Casciaro ${ }^{\mathrm{f}}, \varnothing$. Rideng ${ }^{\mathrm{g}}$, B. Gersak ${ }^{\mathrm{h}}$ \\ ${ }^{a}$ The Intervenional Centre, University of Oslo, Sognsvannsveien 20, 0027 Oslo, NORWAY \\ ${ }^{\mathrm{b}}$ Institute of Computer Graphics and Vision, Graz University of Technology, Inffeldgasse \\ 16/2, 8010 Graz, AUSTRIA \\ ${ }^{\mathrm{c} B i o m e c h a n i c s}$ and Engineering Design Section, Katholieke Universiteit Leuven, Celestijnenlaan \\ 200A, 3001 Heverlee, BELGIUM \\ ${ }^{d}$ Dept. of Industrial Design, Delft University of Technology, Landbergstraat 15, 2628 CE Delft, THE \\ NETHERLANDS \\ ${ }^{\text {e}}$ Siemens Molecular Imaging, 23-38 Hythe Bridge Street, Oxford OX12EP, UK \\ ${ }^{\mathrm{f} S e c t i o n}$ of Lecce, Institute of Clinical Physiology CNR, Campus Ecotekne, Via Lecce-Monteroni, \\ 73100 Lecce, ITALY \\ ${ }^{\mathrm{g}}$ Kongsberg SIM, Bygdøy alle 5, 0257 Oslo, NORWAY \\ ${ }^{\mathrm{h}}$ Dept. of Cardiovascular Sugery, Medical Centre Ljubljana, Zaloska 7, 1000 Ljubljana, SLOVENIA
}

\begin{abstract}
Minimally invasive therapy (MIT) is one of the most important trends in modern medicine. It includes a wide range of therapies in videoscopic surgery and interventional radiology and is performed through small incisions. It reduces hospital stay-time by allowing faster recovery and offers substantially improved cost-effectiveness for the hospital and the society. However, the introduction of MIT has also led to new problems. The manipulation of structures within the body through small incisions reduces dexterity and tactile feedback. It requires a different approach than conventional surgical procedures, since eye-hand co-ordination is not based on direct vision, but more predominantly on image guidance via endoscopes or radiological imaging modalities. ARIS*ER is a multidisciplinary consortium developing a new generation of decision support tools for MIT by augmenting visual and sensorial feedback. We will present tools based on novel concepts in visualization, robotics and haptics providing tailored solutions for a range of clinical applications. Examples from radio-frequency ablation of liver-tumors, laparoscopic liver surgery and minimally invasive cardiac surgery will be presented. Demonstrators were developed with the aim to provide a seamless workflow for the clinical user conducting image-guided therapy.
\end{abstract}

Keywords: Image-guided Therapy, Augmented Reality, Minimally Invasive Therapy

\section{INTRODUCTION}

The ARIS*ER (Augmented Reality in Surgery - European Research network) consortium was founded based on the need to train European researchers capable of developing guidance technologies to support Minimally Invasive Therapy (MIT), which requires cross-disciplinary collaboration between medical science, control science, micro mechanics, human factors and information technologies. The ARIS*ER network brings together these advanced tools and trains a group of young researcher with the required interdisciplinary skills.

The scientific and technological problems involved in advancing MIT are complex and highly multidisciplinary. We have chosen to focus on Augmented Reality as a common theme for the technologies to be employed. Augmented Reality can be defined on the Milgram's virituality continuum [1], which is ranging from the real world (non-modeled reality) to the virtual world ( $100 \%$ modeled reality), as being closer to the real world than the virtual world. Augmented Reality is commonly used as a term describing the visual overlay of computer generated imagery over real world imagery in a spatially and temporally registered fashion. We have chosen to use the term Augmented Reality beyond

Human Vision and Electronic Imaging XIII, edited by Bernice E. Rogowitz, Thrasyvoulos N. Pappas,

Proc. of SPIE-IS\&T Electronic Imaging, SPIE Vol. 6806, 68060K, @ 2008 SPIE-IS\&T · 0277-786X/08/\$18 
visual augmentation and include haptic augmentation, image-registered navigation and combinations of these as alternative forms of augmenting reality. A premium is put on improving eye-hand co-ordination and thus enhancing the accuracy of MIT procedures, improving the margin of safety, and enlarge the potential applications for MIT. The current key limiting factors in MIT are:

- immature and unreliable tools for real-time 3D-navigation

- fragmented research (geographically and disciplinary) prevents rapid development

- lack of researchers capable to operate across the integrated fields of 3D visualization, image processing, robotics and radiology, while having sufficient insight into the workflow, clinical tasks and human factors relevant for interventional radiology and minimally invasive surgery.

The ARIS*ER consortium is a EU funded Marie Curie Research Training Network established to address these bottlenecks.

Computer aided image-guided 3D-navigation tools should be improved to reach the level of (and preferably, exceeding that of) the 3D-navigation currently based on direct vision and direct "feel". We have developed new software navigation tools for MIT based on intelligent processing and presentation of fused image information integrated in workflow oriented decision support tools. We have also developed concepts showing how intra-operative image-guidance can be cross-linked with robotic systems and haptic devices to further facilitate multi-modal interaction.

A collection of clinical application areas was identified in the early phase of this project. These applications have been studied and targeted for development of new technological solutions with high potential added value.

\subsection{MRI-guided RF ablation}

Hyperthermia ablation is used for treatment of benign and malignant tumours. The tissue response to heating is related to the absolute temperature induced in the tissue and exposure time. Irreversible tissue destruction occurs when tissue is exposed to temperatures in excess of $50-55^{\circ} \mathrm{C}[2,3]$. Hyperthermia ablation has been widely used for treating malignant liver tumors not amenable to conventional surgical resection. Heating modalities in clinical use include laser, microwave, radiofrequency, and focused ultrasound [4]. The exact size and shape of the resulting ablation is difficult to predict because of heterogeneous distribution of the thermal energy due to local disparity in tissue composition, tissue anatomy, and vascularization. In clinical series, local tumor progression after ablation therapy has been reported to be as high as $30-60 \%$, indicating the presence of viable malignant cells at the treated site [5]. Improved intra procedural monitoring may reduce the local tumor progression rates associated with thermal ablation and prove beneficial in regard to longterm patient survival [6].

\subsection{Laparoscopic liver resection}

Laparoscopic liver resection was first reported in 1991. Since then, more than 500 journal articles have been published describing the full spectrum of resections of benign and malignant liver and pancreatic disease [7]. Leaders in the field believe that there will be a significant role for minimally invasive approaches to hepatic surgery in the future [8]. One of the key issues identified by ARIS*ER experts related to this clinical application was reduction of bleedings. This issue was also raised by Optiz where it is concluded that bleeding remains a major complication during laparoscopic surgery [9], other reports support the same conclusion [10]. Another key issue identified by ARIS*ER experts relates to visualization which should support navigation and orientation. Such visualization can be accomplished by fusion of MRI, CT or ultrasound images with video-images. Bao has reported on a system for ultrasound-to-computer-tomography registration for image-guided laparoscopic liver surgery [11]. The results were acquired using a phantom, assuming a rigid body situation. It provides an interesting background for the work in ARIS*ER. Other reports describe specific issues related to navigation accuracy and projection accuracy (for Augmented Reality) in the domain of laparoscopic liver resection $[12,13,14,15]$.

\subsection{Endoscopic mitral valve repair/replacement}

The introduction of minimally invasive cardiac surgery has lead to reduced hospital stay and shorter recovery. Endoscopic mitral valve surgery can now be routinely performed through a $4 \mathrm{~cm}$ working port $[17,18,19,20,21]$, usually in the $4^{\text {th }}$ intercostal space. The procedure has also been performed by several groups using the daVinci robot system $[22,23]$. 
As in the open chest cardiac surgery procedure, in the Minimally Invasive procedure the heart must be stopped and for this, the patient must be placed under Cardio Pulmonary Bypass (CPB). One of the steps for implementing CPB is the sectioning of the Ascending Aorta in order to isolate the heart and the coronary arteries from the rest of the arterial circulation. During open surgery this sectioning is done by using a clamp on the artery. Its use is widespread and it is easy to place, as during a normal procedure access to the aorta is easy. In endoscopic mitral valve surgery this can also be done, but requires an extra surgical port. A device such as the Endoclamp ${ }^{\mathrm{TM}}$ Balloon Catheter provided by Heartport (Johnson \& Johnson) can be used to perform the sectioning of the aorta internally, without extra port. The device consists of a catheter, with an inflatable balloon in the distal part that once inflated in the correct location will provide the required isolation from the heart to the rest of the circulation. This device is used nowadays to perform MIS with success. However, the use of the device discussed before presents some technical difficulties, which are mostly related with placement and monitoring during the surgery. It is extremely important to guarantee its correct placement at all times. Failure to place the balloon correctly has the potential of leading to adverse events. Consequently the balloon position is subject to continuous monitoring. We have studied how computer-guided methods could possibly improve this key aspect of the procedure.

\section{USER CENTERED DESIGN}

There is a sound medical and economic incentive for appropriate user-centric system design. Designs that consider human abilities and adaptability will be more effective and result in higher productivity. This applies to the medical domain and especially to the design of modern operating theaters. The rapid advancement of new information and imaging technology has provided a wealth of pre-operative and intra-operative (imaging) patient data. When properly used, this information may support the surgical procedure, improve efficiency, safety and cost effectiveness compared to existing clinical procedures and may even allow the development of novel procedures that could not be performed before. The designer is faced with the challenge, to define the relationship between the surgeon and the information system within the appropriate context of performing an operation in the operating room. While making the information more transparent, multiple issues related to expert knowledge, medical workflow, patient safety, machine cognition including feedback and decision making need to be addressed. Furthermore, the designer play a critically important role to bridge the language and culture barriers between medical doctors on the one hand versus technology specialists on the other hand, so that all stakeholders can discuss the design process on a common communication and working platform.

\subsection{Workflow assessment and analysis (adapted from Parmar 2007 [24])}

Medical workspace is a composite web of experts and expert systems that need to be addressed before making an attempt to suggest solutions which offer increased accuracy of the medical procedure. This requires a sound analysis of the complex medical world which is governed by various levels of decision making. To address the complexity of the medical world demanded the deployment of a variety of techniques and strong intellectual and methodological discipline, not a commitment to the hegemony of a single research modality. Currently, there exist no single route of domain specific design ethodologies to approach the complex task, but richer possibilities were found in integrating and customizing methods from domain like social research and design research. To match the project needs customizing and integrating parts of selected user observation methods in the user centered design process was found to be very useful and complementary. We involved medical experts and technologist as a co-designers at various stages of the product development cycle by adapting a standard social science research method called participatory action research [25] (in which some people of the organization, understand and participate actively with the professional researchers thought the research process from initial design to the presentation of the results and discussions).

We have developed a method for assesing requirements and possibilities through user observations and structuring them in a workflow integration matrix [26]. We have based our method on the standard design cycle and added some components of the method which are pointed below:

- User observations: In order to investigate the information lacunas and user requirements and have a understating of the surgical workflow we integrated 6 different methods of user observation. In total we followed 22 surgical observations, 15 expert interviews, 3 focus groups with 21 surgeons and radiologist, 8 
technologist interviews, 40 days stay in the hospital spanning over 6 countries were conducted. Specific user requirements and results from focus groups are forthcoming in a publication.

- Workflow integration matrix: In order to analyze and integrate the user findings from the above method common communication platform in the form of "workflow integration matrix" was formulated. Apart from capturing a composite overview of the current surgical workflow it also helped in defining the user requirements in a systematic way, so that they can be traced back to the key problem issues. As this matrix was a new approach, it was tested for its communicability with the technology partners, researchers and medical doctors in the consortium. Few of the findings thus generated have been taken up as research areas by the technology researchers in the consortium in their respective domains. These findings are also assisting some of the medical and technical partners to visualize the future medical workflow limitations and possibilities.

\subsection{Task analysis (adapted from Studeli 2007 [27,28])}

We have developed a framework tailored to the design and future MIT computer systems. It addresses aspects of usercentered design, information presentation, surgeon workload and safety

- Task analysis (ISO 13407) and matching tasks with roles, ISO 6385, is the first element of the evaluation framework and should be performed in a co-research setting with technologists, experienced surgeons and a Human Factors (HF) specialist moderating the sessions. A big sheet (A0) with grid can be used during a workshop, depicting the main tasks of the surgical procedure in columns (inspired by Jalote-Parmar et al. 2006) and roles of the team and the equipment of the system in rows. Different tasks and subtasks of the medical procedure are to be identified and described. Subsequently descriptions of the subtasks for the different roles are to be recorded in the appropriate cells (roles as defined by van der Veer et al 2002).

- Next, an adapted critical incidents analysis (ISO/TR 16982) should be performed. The surgical procedure should be presented and explained by surgeons, following the mapping into the large grid (above). Participants will select critical subtasks and mark the corresponding cell on the grid with colour coded stickers. Safety critical subtasks are marked red, subtasks with critical lack of information are marked blue and subtasks with critical workload yellow. The HF moderator asks the participants to explain their choice. The result is later analyzed and forms the questions for the following parallel steps, the interviews on the selected key requirements in ergonomics.

- Interviews (ISO/TR 16982) with experienced medical doctors in MIT: the subjects are questioned individually to deepen the understanding of the outcome from the critical incidents analysis in order to identify the tasks with especially high demands on information, especially high workload situation, and especially high risk for the patients' safety.

- Future technical possibilities for key tasks are discussed with focus on visualization, medical aspects and robotics.

- Results from all workshops and interviews are analyzed and combined to generate hypotheses on the design of future MIT computer systems.

This evaluation framework starting with a task analysis and ending with creating hypothesis on the design of future systems takes in account major advances in technology during the design process. Our question also looks ahead: How to evaluate not yet existing technology? How can we ensure usability of our computer system, which takes major breakthroughs in technology in account? The benefit of looking into the future and describing future possibilities is that more precise and beneficial input for actual running system of the future can be given.

\section{TECHNOLOGICAL BUILDING BLOCKS}

A number of key technological problems were defined early in the project which would need to be addressed in order to provide essential technological building blocks. This part of the project was executed in a bottom-up fashion, while the higher level demonstrator and application development was performed in an top-down fashion based on the requirements defined during the user-centered co-design work. 


\subsection{Segmentation of the liver from CT images (adapted from Massoptier 2007 [29])}

Liver interventions have to be carefully planned to make sure that the critical structures and pathological regions are spatially localized. The planning must be supported by accurate computerized segmentation and registration tools. The segmentation will delineate the liver and localize precisely tumors inside it, distinguishing healthy tissue from pathological and identify vascular architectures. In Radio Frequency (RF) ablation planning, safety margins are added around tumors in order to ensure adequate treatment of the target volume. Also in surgical interventions, the same concept is expressed as a resection margin (typically $1 \mathrm{~cm}$ ) added to ensure that also possible infiltrating tumor cells are removed. This margin's size is a crucial point. If the margins are too small, there is a risk of a leaving cancerous regions untreated. Unnecessarily large margins may cause side effects [30], as ablation or resection of too large areas of the organs and/or important vessels of the organ vasculature. Automatic liver segmentation is a challenging task because the liver shares image intensity values with other close organs (kidneys for example). Moreover their boundaries are not generally sharp. An algorithm as developed to address these challenges:

First, a 3D mean shift filter is used to homogenize image intensity values of each different structure by keeping clear and sharp edges. Then, we use an adaptive threshold by calculating simple statistics in order to segment the target organ (i.e. the liver). This step is followed by classical image processing techniques in order to isolate the liver. Finally, a gradient vector flow (GVF) active contour method is applied to obtain a smooth contour of the liver.

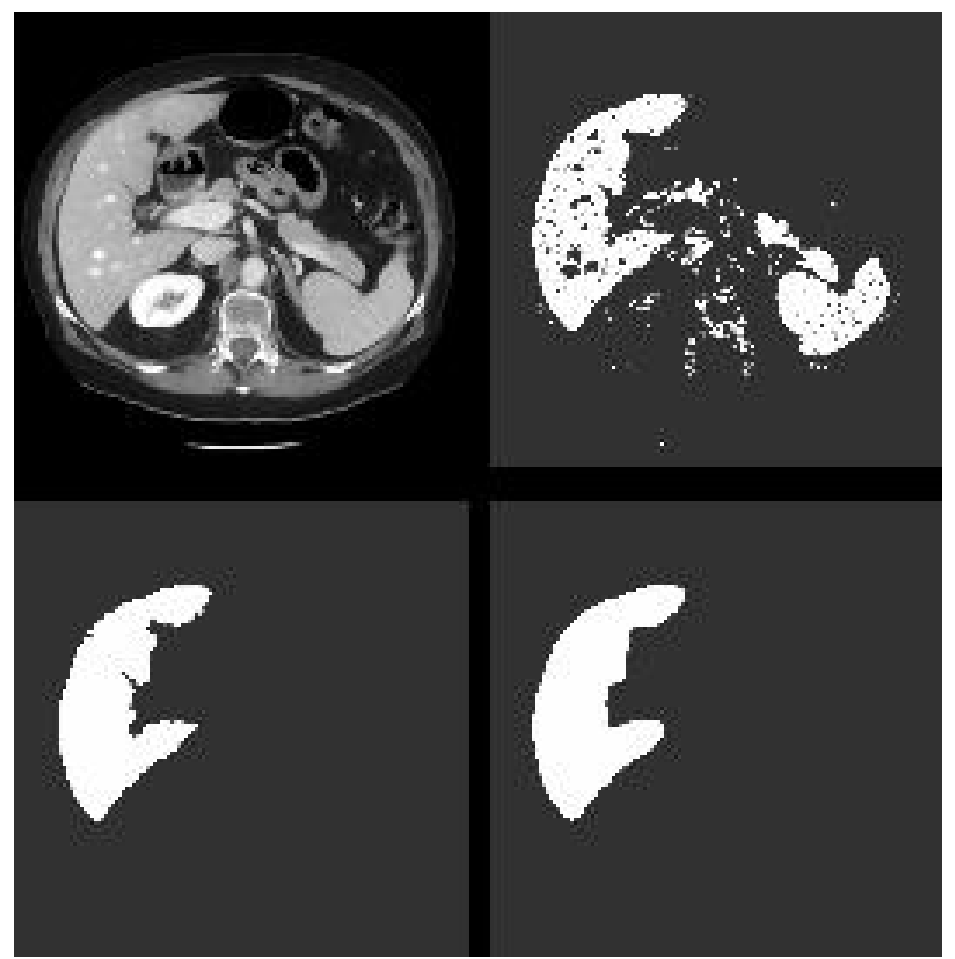

Fig. 1. Upper left: Mean shift filtered CT image. Upper right: Result of adaptive threshold. Lower left: After the labeling and the hole filling steps. Lower right: Result of the GVF active contour. From [29]

To quantify the segmentation result the DSC was calculated between the automatically segmented images and manually segmented images. The DSC was found to be between 0.93 and 0.98 independently from CT or MRI. By using the median filter instead of the mean shift filter, we achieved a DSC index between 0.91 and 0.95 . The processing time was found to be 4.5 s for a $256 \times 256$ image matrix. 


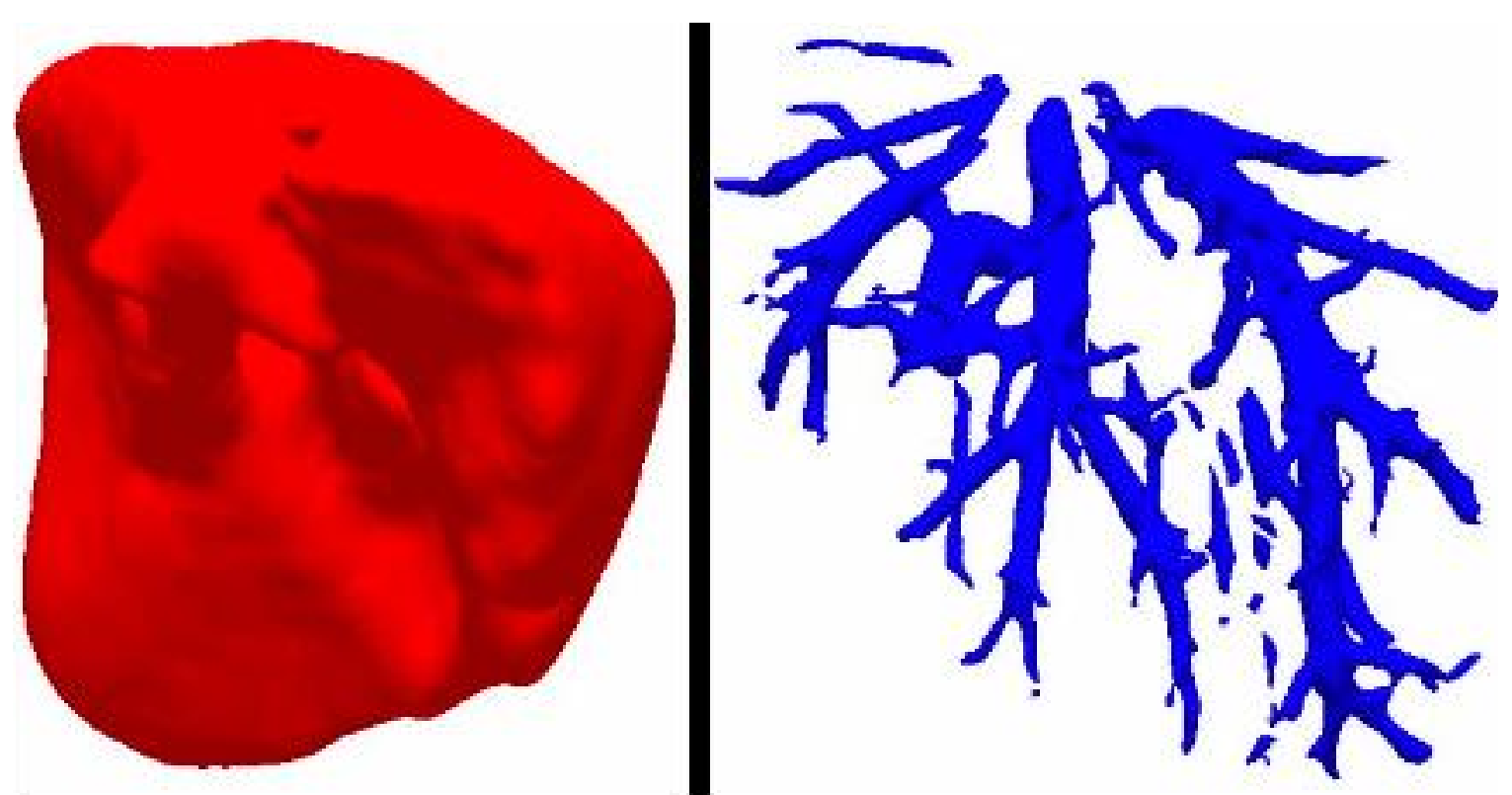

Fig. 2. 3D visualization of the segmented liver as well as the vessel tree using a tube filter employing calculation of the Hessian matrix in each voxel and calculating vesselness based on eigenvalue ratios. From [29]

\subsection{Context visualization for augmented reality (adapted from Kalkofen 2007 [31])}

The concept of augmented reality is commonly understood as augmenting visual impressions based on visible light (direct vision or real-time video streams) with context relevant computer generated imagery. The task of carefully overlaying synthetic data on top of the real world imagery is not trivial. We have addressed the challenge of taking into account the information that is about to be occluded by our augmentations as well as the visual complexity of the computer-generated augmentations added to the view. Our work is inspired by Focus and Context $(\mathrm{F}+\mathrm{C})$ visualization techniques, a powerful instrument for visually communicating relevant structures. To address the problem of augmentations occluding useful real imagery, we introduce the notion of context preserving X-Ray vision which controls the removal of real world information based on an importance measure of this information. Moreover, we address the problem of cluttered displays by providing solutions for controlling depth complexity with an interactive, user controlled filter which combines spatial and contextual information. The techniques that were developed are embedded in a novel AR visualization framework which operates on an intermediate representation extracted from conventional 3D models. This representation is image based and can be described as a sparse, non-uniformly sampled, view-dependent volumetric model. We use recent GPU programming techniques for efficient rendering of this representation inspired by the G2 buffer framework [32].

Fig. 3 shows the problem of overlaying real-world imagery with synthetic data, as the synthetic data appears to be closer to the viewer than the skin due to the non-intended occlusion depth cue (even when rendered transparently). This problem may be solved with edges extracted from the real video stream. The extracted edges provide an additional depth cue and since they come from the real imagery, they are also able to preserve important landmarks (such as the entry points). 

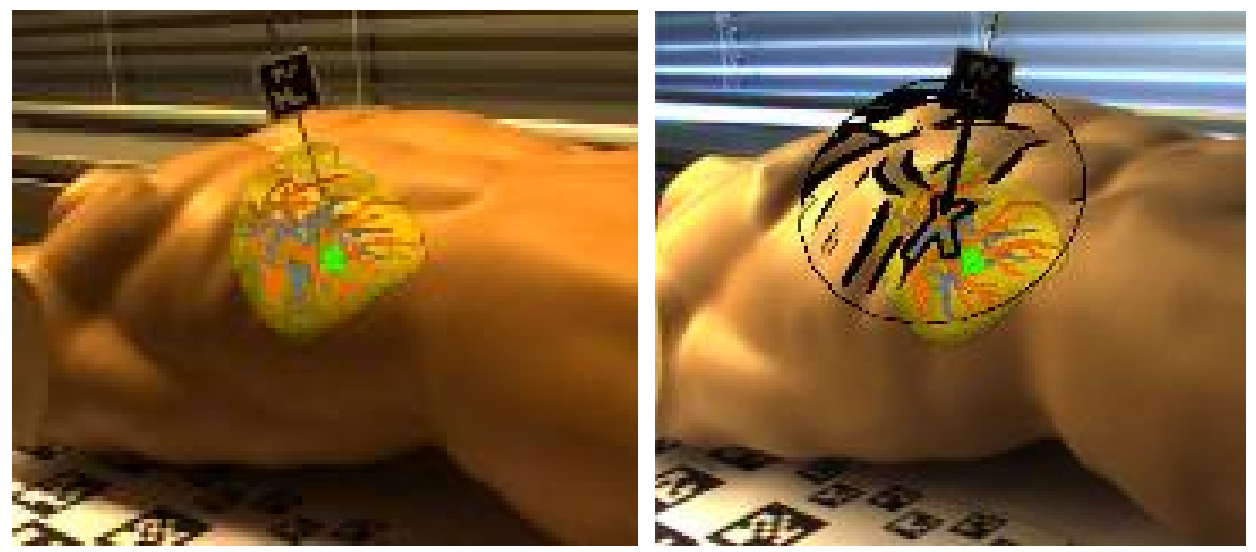

Fig. 3. By enhancing with edges, extracted from the video stream we provide not only important occlusions but also we preserve important landmarks. The area where edges are being detected is interactively controlled by a flat Magic Lens which locally restricts augmentations with edges and prevents from cluttered displays. From [31]

\subsection{Software framework for augmented reality in surgical procedures (adapted from Kalkofen 2006 [33])}

We have developed a software framework for augmented reality visualization in surgical procedures called Studierstube Medical. Studierstube Medical is based on Studierstube framework [34], an AR framework which has now been under development for 10 years. The medical version of Studierstube features enhanced modularity and extends Studiersube with a number of components specifically targeted at developing medical software applications such as LiverPlanner $[35,36,37]$. Studiestube Medical was designed with the following requirements in mind:

- $\quad$ Real-time data acquisition In contrast to modalities such as CT or MR, which are normally not acquired in real time, AR applications require the management of streaming input data such as tracking, ultrasound imaging or video data. The handling of such data requires real-time algorithms and also careful synchronization, in particular between simultaneously acquired data from heterogeneous sources, which must be correlated. For example, consider simultaneous acquisition of endoscopic video and US images with tracked probes. The data streaming must also be flexible enough to permit reuse of the solutions across different applications. We have developed a software architecture for streaming data based on a pipes- and-filters pattern [Error! Reference source not found.], which can fulfill these requirements and provides specific support for tracking and video data.

- Consistent data models The requirements of surgical planning, navigation and simulation go beyond the simple display of volumetric data acquired in previous image scans. For sophisticated computer-aided medicine, anatomical and pathological structures must be explicitly modeled and manipulated. Diverse application requirements make multiple redundant structures necessary - for example, the same sampled volumetric dataset may be used to compute a surface model of an organ, a graph model of the contained vessel structure and a polyhedral model of internal tissue. Physicians demand predictable and reproducible results, so all these representations must be kept in a consistent state throughout the medical workflow while permitting arbitrary operations on the data.

- Visualization Compared to conventional screen-based navigation, AR has elevated requirements for visualization. It should support a wide variety of display devices with very specific properties, such as tracked head-mounted display (HMD), stereoscopic or video-see through configurations. The actual visualization techniques must be mostly independent of the viewing mode and display type, and must be able to simultaneously display all kinds of data models in real time, for example scanned volumetric images with direct volume rendering together with polygonal rendering of segmented surface models. The requirements lead us to a design of a component-based system.

\subsection{Haptic feedback in minimally invasive surgery (adapted from Sette 2007 [39])}

Despite the benefits of Minimally invasive surgery, many surgeons find it difficult to manually perform procedures that require dexterity and fine motion capability with minimally invasive techniques. Remotely controlled robotic 
manipulators have been developed to increase dexterity and accuracy. Commercially available systems have some distinct drawbacks, such as: (i) lack of local manipulability of the tools, (ii) the absence of force and tactile feedback to the surgeon's hands and (iii) lack of a natural interface with the surgeon. It would be useful if the surgeon would have the same freedom to acquire information as he/she has in open surgery. By rubbing and palpating the tissue he/she gets information on texture and structure of the tissue, on blood stream pulsations through arteries, etc. We are aiming for restoring this kind of feeling during the robotic surgery, thus introducing augmented reality beyond visual augmentation.

A teletactile system consists of three basic elements: (i) a tactile sensor, to remotely collect raw tactile data, (ii) a tactiledata processing system, and (iii) a 'tactile display', presenting the tactile information in a natural and realistic way to the surgeon. A telesurgical system with reliable force feedback can extend the application range of minimally invasive surgery to more complex procedures. For medical decisions that are primarily based on touch information, such as the localisation of a tumour or hidden arteries or the quality verification of a suture, surgeons mainly rely on their stiffness perception and discrimination abilities.
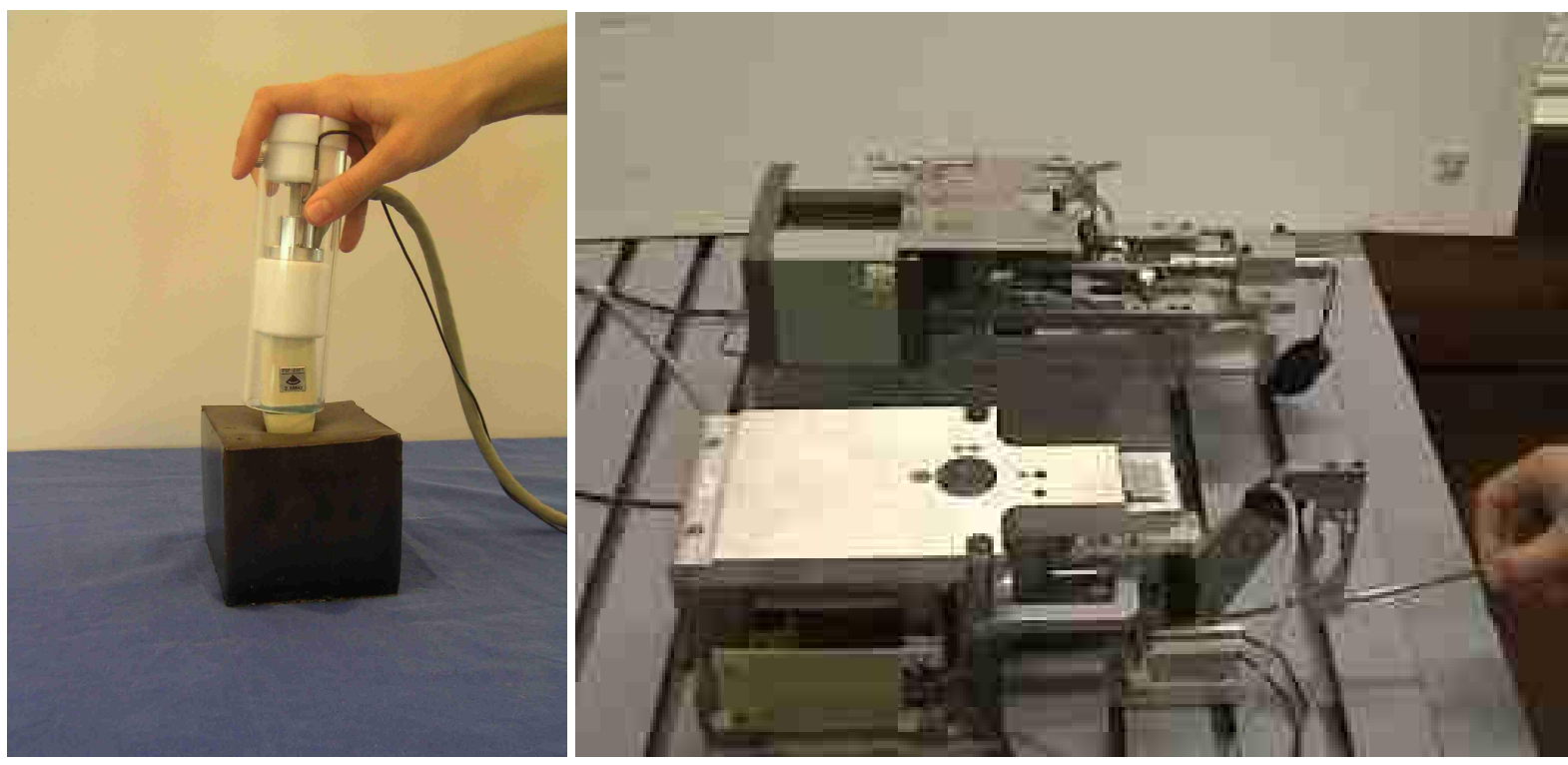

Fig. 4. The left image shows a prototype tactile sensor based on elastography. The right image shows a prototype teleoperation system capable of reproducing force feedback. From [39].

An alternative way to develop a tactile sensor is to use the elastography. Elastography is a method that can ultimately generate a new kind of image called elastograms. These images have the characteristic of been based on the stiffness of the organs. This is a new powerful way of diagnosis, for example a tumour will have a different stiffness of the surrounding tissue, this property can be easily identify with the elastography. This new idea can be applied also to the haptic feedback, a sensor based on elastography can record information on the stiffness of tissues and give to the surgeon these information in a "tactile way". A system was developed for the calculation of the stiffness of some volumes when the elastogram is given. The system was tested on four different phantoms with four different stiffnesses, these first experiments had shown a very good behavior when used on homogeneous volumes. 


\section{DEMONSTRATOR}

\subsection{Demonstration application supporting RFA ablation of liver tumors}

Using the Studierstube Medical framework a demonstrator application was developed to support percutanous placement of probes for RF ablation of liver tumors. The software application that was implemented consists of three modules supporting each of the major phases of the procedure: pre-, intra- and post-operative.

\section{Pre-Operative and post-operative module}

This module supports the pre-operative exploration of pre-operative CT/MR volumetric images in addition to 3-D views of segmented structures in relation 2-D slices of the volumetric images. After surgery it is important to validate the results. Hence, the module supports fusion of pre-operative and post-operative CT/MR images to assess the effect of the ablation.

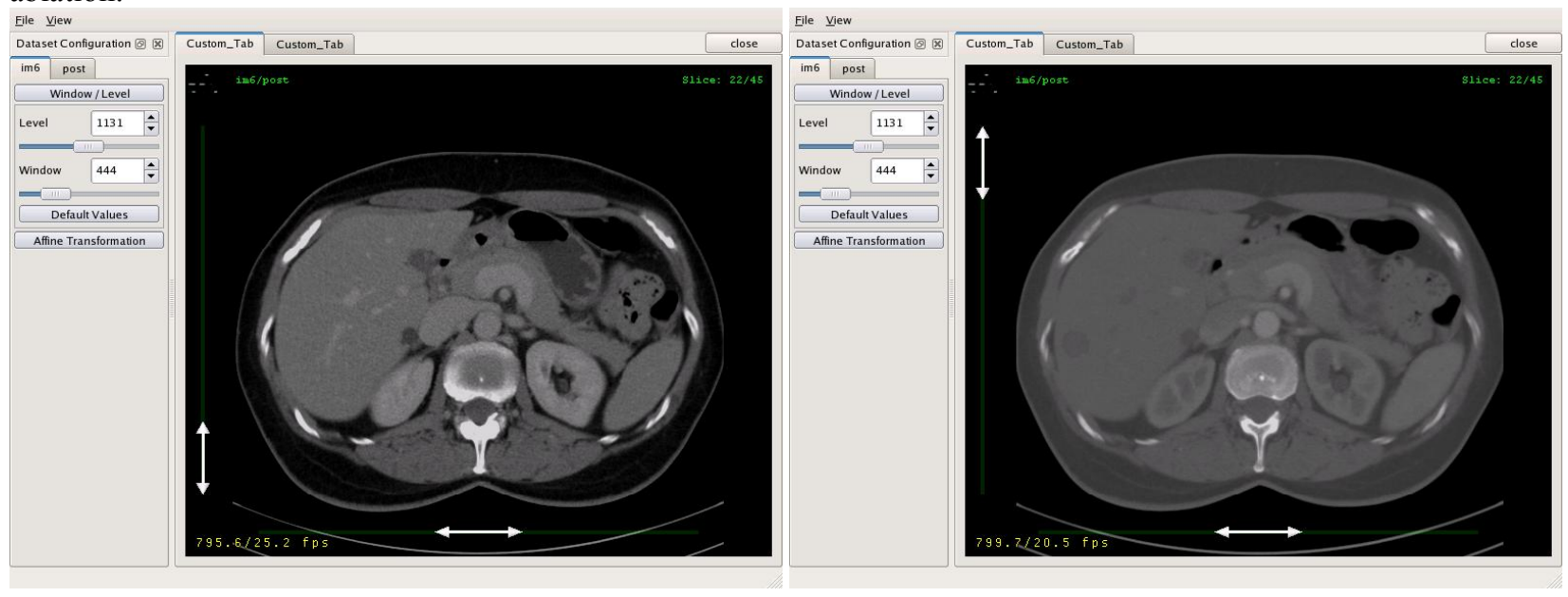

Fig. 5. The left image shows the post-operative module where the pre-operative image is visualized. The right image shows the post-operative image. A seamless transition between pre-operative and post-operative images can be achieved by the use of a transparency slider.

\section{Intra-Operative Module}

The intra-operative module of the RFA demonstrator gives the clinical user a supportive interaction by the fusion of relevant information visualized in a head-mounted display and registered accurately with the patient Fig. 6.

\section{DISCUSSION}

Minimally invasive therapy is raising expectations among the public and efforts such as the ASRIS*ER project will help doctors to deliver a higher quality of care with reduced operative morbidity. A number of meta-studies, derived to provide arguments for evidence-based medicine, have shown that MIT does reduce hospital stay and post-operative pain and that it allows faster return to normal activity. Examples of such studies, extracted from the Cochrane library are:

- Systematic review: Laparoscopic versus open surgery for suspected appendicitis [40]

- Economic evaluation database: A randomized, controlled, clinical study of laparoscopic vs open tension-free inguinal hernia repair [41]

- Economic evaluation database: Feasibility of 23-hour hospitalization after laparoscopic fundoplication [42] 


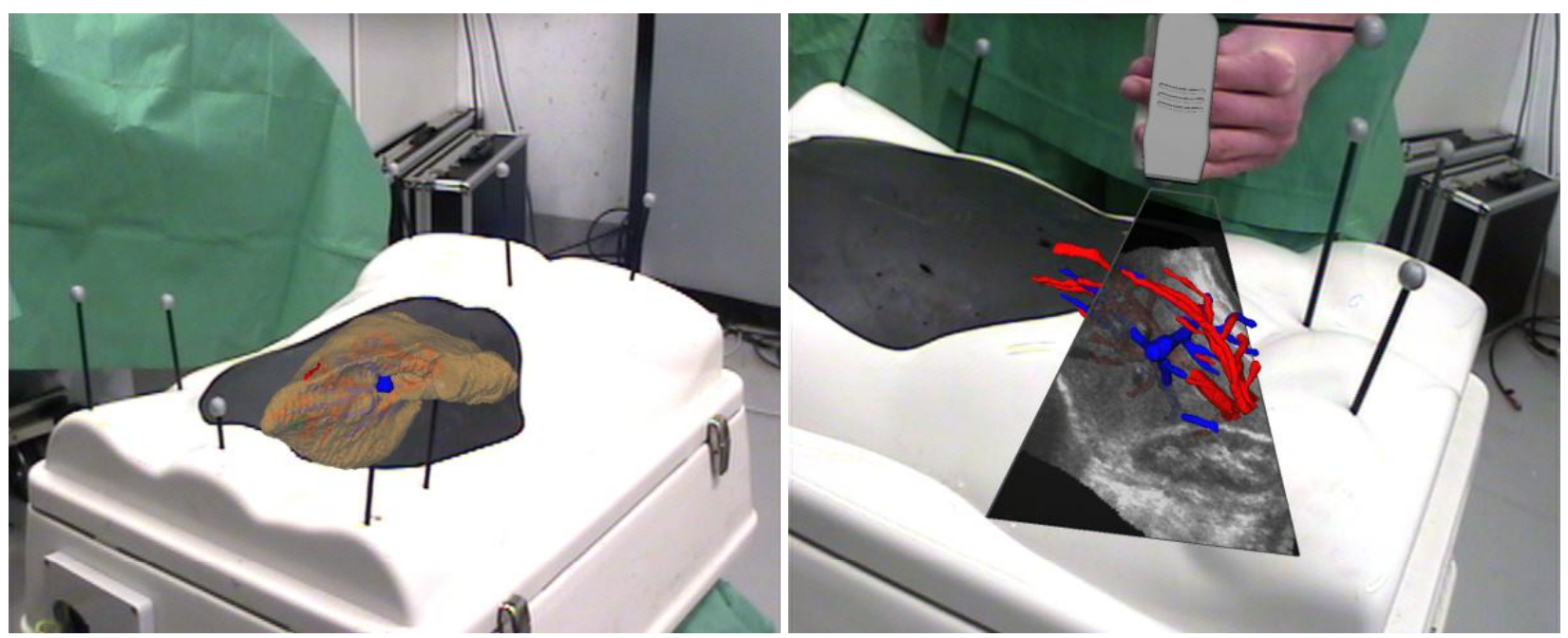

Fig. 6. The left images shows the hepatic and portal vessels, tumor and liver overlayed on the phantom. All structures are color coded. The right image demonstrates have the system allows fusion of the intra-operative ultrasound images with segmented structures from pre-operative images. (In this case the vessel structure).

Some of these studies show higher complication rates with minimally invasive techniques compared to open techniques. This suggests that the indications for MIT could be improved and that the intra-operative support is inadequate. This latter is reinforced in that some reviewers comment that the work reported is by experienced surgeons and that results might be different with less experienced operators. Currently $30 \%$ of all surgical procedures are performed under minimally invasive conditions according to Cambridge Consultants. New developments of 3D navigation tools, designed with human factors in mind, offers the potential for considerable improvement and in doing so addresses some of the main societal problems relating to healthcare. The improvements will relate to:

- Increased quality of minimally invasive therapy

- Increase utilisation of minimally invasive therapy

- Reduction of health care costs

The multidisciplinary ARIS*ER project has brought together competences in a wide variety of fields, ranging from cognitive sciences to design and engineering sciences, applied mathematics and the science of medicine. The consortium was constituted to create interactions across the whole value-chain from fundamental science through development and use. Carrying out work in such a multidisciplinary team have resulted in unforeseen synergies. Existing institutional and disciplinary boundaries related to working culture, such as between clinicians and engineers, was sought bridged. We have presented results forged from cross-disciplinarity and integration along several axes:

- Activity integration between medical practice and informatics technology

- Horizontal integration by drawing together those involved in research and development in the fields of a variety of digitally operated medical devices and health care provision. ${ }^{1}$

- Vertical integration by including actors in the whole value chain; academic researchers, industrial players and clinical users

- Cross-sectoral integration by working apposition of both small and large industrial concerns with a number of active academic centers.

\section{ACKNOWLEDGEMENTS}

This work was funded by the European Union through the Marie Curie grant MCRTN-CT-512400. The authors would like to thank all of the early-stage and experienced researchers recruited through the contract: Tangui Morvan, Jordi Cornella, Denis Kalkofen, Judith Muehl, Mauro Sette, Ashis Jalote-Parmar, Thomas Studeli, Sergiy Milko, Pablo Lamata de la Orden, Laurent Massoptier, Perrine Paul, Piotr Makowski, Tamer Fahmy, Hugo Furtado and Morita Terumasa. Gratitude is also expressed to the external and internal experts and collaborators who have given an invaluabe contribution. 


\section{REFERENCES}

1 P. Milgram and A.F. Kishino, Taxonomy of Mixed Reality Visual Displays, IECE Transactions on Information and Systems, E77-D(12), pp. 1321-1329, 1994

2 Goldberg SN, Gazelle GS, Mueller PR. Thermal ablation therapy for focal malignancy: A unified approach to underlying principles, techniques, and diagnostic imaging guidance. American Journal of Roentgenology 2000; 174(2):323-331.

3 Storm FK, Harrison WH, Elliott RS, Morton DL. Hyperthermic Therapy for Human Neoplasms - Thermal Death Time. Cancer 1980; 46(8):1849-1854.

4 Vogl TJ, Straub R, Eichler K, Sollner O, Mack MG. Colorectal carcinoma metastases in liver: Laser-induced interstitial thermotherapy - Local tumor control rate and survival data. Radiology 2004; 230(2):450-458.

5 Solbiati L, Livraghi T, Goldberg SN, Ierace T, Meloni F, Dellanoce M et al. Percutaneous radio-frequency ablation of hepatic metastases from colorectal cancer: Long-term results in 117 patients. Radiology 2001; 221(1):159-166.

6 Wood TF, Rose DM, Chung M, Allegra DP, Foshag LJ, Bilchik AJ. Radiofrequency ablatian of 231 unresectable hepatic tumors: Indications, limitations, and complications. Annals of Surgical Oncology 2000; 7(8):593-600.

7 Hansen PD. Laparoscopic resections of liver and pancreas. Journal of Gastrointestinal Surgery 2004; 8(8):925-926.

8 Mala T, Edwin B, Gladhaug I, Fosse E, Soreide O, Bergan A et al. A comparative study of the short-term outcome following open and laparoscopic liver resection of colorectal metastases. Surgical Endoscopy and Other Interventional Techniques 2002; 16(7):1059-1063.

9 Opitz I, Gantert W, Giger U, Kocher T, Krahenbuhl L. Bleeding remains a major complication during laparoscopic surgery: analysis of the SALTS database. Langenbecks Arch Surg 2005 Feb 8.

10 Roviaro GC, Varoli F, Saguatti L, Vergani C, Maciocco M, Scarduelli A. Major vascular injuries in laparoscopic surgery - Still of interest? Surgical Endoscopy and Other Interventional Techniques 2002; 16(8):1192-1196.

11 Bao P, Warmath J, Galloway R, Jr., Herline A. Ultrasound-to-computer-tomography registration for image-guided laparoscopic liver surgery. Surg Endosc 2005 Jan 10.

12 Herline A, Stefansic JD, Debelak J, Galloway RL, Chapman WC. Technical advances toward interactive imageguided laparoscopic surgery. Surgical Endoscopy-Ultrasound and Interventional Techniques 2000; 14(7):675-679.

13 Herline AJ, Herring JL, Stefansic JD, Chapman WC, Galloway RL, Dawant BM. Surface registration for use in interactive image-guided liver surgery. Medical Image Computing and Computer-Assisted Intervention, Miccai'99, Proceedings 1999; 1679:892-899.

14 Herline AJ, Herring JL, Stefansic JD, Galloway RL, Dawant BM, Pinson WC et al. Technology assisted liver surgery using image fusion. Hepatology 1999; 30(4):162A.

15 Herline AJ, Stefansic JD, Debelak JP, Hartmann SL, Pinson CW, Galloway RL et al. Image-guided surgery Preliminary feasibility studies of frameless stereotactic liver surgery. Archives of Surgery 1999; 134(6):644-649

16 Stefansic JD, Herline AJ, Shyr Y, Chapman WC, Fitzpatrick JM, Dawant BM et al. Registration of physical space to laparoscopic image space for use in minimally invasive hepatic surgery. Ieee Transactions on Medical Imaging 2000; 19(10):1012-1023.

17 Casselman FP, Van Slycke S, Wellens F, De Geest R, Degrieck I, Van Praet F et al. Mitral valve surgery can now routinely be performed endoscopically. Circulation 2002; 106(19):555.

18 Gersak B, Sutlic Z. Aortic and mitral valve surgery on the beating heart is lowering cardiopulmonary bypass and aortic cross clamp time. Heart Surgery Forum 2002; 5(2):182-U32.

19 Gersak B. A technique for aortic valve replacement on the beating heart with continuous retrograde coronary sinus perfusion with warm oxygenated blood. Annals of Thoracic Surgery 2003; 76(4):1312-1314.

20 Gersak B, Sostaric M, Kalisnik JM. Endoscopic aortic valve replacement. Heart Surgery Forum 2003; 6(6):E197E199.

21 Saunders PC, Grossi EA, Sharony R, Schwartz CF, Ribakove GH, Culliford AT et al. Minimally invasive technology for mitral valve surgery via left thoracotomy: Experience with forty cases. Journal of Thoracic and Cardiovascular Surgery 2004; 127(4):1026-1032.

22 Kypson AP, Chitwood WR. Robotic mitral valve surgery. American Journal of Surgery 2004; 188(4A):83S-88S.

23 Bolotin G, Kypson A, Nifong LW, Reade C, Freund W, Chitwood WR. Robot-assisted mitral valve repair with microwave atrial fibrillation ablations: early clinical results. Journal of the American College of Surgeons 2003; 197(3):S24-S25. 
24 A. Jalote-Parmar, P.M.T. Pattynama, R.H.M. Groossens, A. Freudenthal, E. Samset, H. de Ridder, Bridging the Gap: A User Centered Design Approach Towards Developing Technological Solutions, in "Novel Technologies for Minimally Invasive Therapies", Lupiensis Biomedical Publications, ISBN 978-88-902880-0-5, 2007, page 93-101

25 Whyte F.W. Participatory action research. Sage publications. America. 1991.

26 Jalote-Parmar A, Pattynama PMT, Goossens RHM, Freudenthal A, Samset E, De Ridder H. Exploring a user centric methodology to investigate and integrate information gathered during medical intervention. In Proceedings of the 16th World Congress on Ergonomics, Maastricht, The Netherlands: International Ergonomics Association. 2006.

27 Stüdeli T., Freudenthal, A., and Samset, E. 2007: Assessing Quality of Computer Systems and User Interfaces of Future Medical Workplaces - Augmented Reality for Minimally Invasive Therapy, In: Y. Sandblad \& $\AA$. Walldius (Eds.) Proceedings of the UITQ 2007 Workshop, May 20 2007, Stockholm, Sweden.

28 Stüdeli, T., Freudenthal, A., de Ridder, H. 2007: Evaluation Framework of Ergonomic Requirements for Iterative Design Development of Computer Systems and their User Interfaces for Minimal Invasive Therapy, In: Allan Toomingas, Ann Lantz and Tomas Berns (Eds.) Proceedings WWCS 2007 Computing Systems for Human Benefits from the 8th International Conference on Work With Computing Systems. May 21-24 2007, Stockholm, Sweden

29 L. Massoptier, S. Casciaro, Segmentatino of Liver Anatomy and Pathology, in "Novel Technologies for Minimally Invasive Therapies", Lupiensis Biomedical Publications, ISBN 978-88-902880-0-5, 2007, page 57-66

30 K. Wurstbauer, H. Deutschmann, P. Kopp, F. Sedlmayer, Radiotherapy planning for lung cancer: slow CT allow the drawing of tighter margins, Radiother Oncol. 2005; 75: 165-170

31 Kalkofen D, Mendez E, Schmalstieg D: "Interactive Focus and Context Visualization in Augmented Reality" Proc. 6th IEEE International Symposium on Mixed and Augmented Reality (ISMAR'07), pp. 191-200, Nara, Japan, October 2007

32 Eissele M., Weiskopf D., and T. Ertl. “The G2-Buffer Framework“, In proceedings of SimVis, 2004, pp. 287-298

33 Kalkofen D, Reitinger P, Risholm P, Bornik A, Beichel R, Schmalstieg D, Samset E, "Integrated Medical Workflow for Augmented Reality Applications", International Workshop on Augmented environments for Medical Imaging and Computer-aided Surgety (AMI-ARCS), Copenhagen, Oct 2006

34 D. Schmalstieg, A. Fuhrmann, G. Hesina, Z. Szalav `ari, L. M. En carnac ao, M. Gervautz, and W. Purgathofer. The studierstube augmented reality project.Presence:Teleoper.VirtualEnviron.,11(1):33-54,2002.

35 A. Bornik, R. Beichel, B. Reitinger, G. Gotschuli, E. Sorantin, F. Leberl, and M. Sonka. Computer aided liver surgery planning: An augmented reality approach. In Robert L. Galloway, editor, Medical Imaging 2003: Visualization and Display,ProceedingsofSPIE,vol-ume 5029. SPIE, SPIE Press, May 2003.

36 A. Bornik, R. Beichel, E. Kruijff, B. Reitinger, and D. Schmalstieg. A hybrid user interface for manipulation of volumetric medical data. In Proceedings of IEEE Symposium on 3D User Interfaces 2006. IEEE,

37 B. Reitinger. Virtual Liver Surgery Planning: Simulation of Resections using Virtual Reality Techniques. PhD thesis, Graz University of Technology, 2005.

38 Frank Buschmann, Regine Meunier, Hans Rohnert, Peter Sommerlad, and Michael Stal. Pattern-oriented software architecture: a system of patterns. John Wiley \& Sons, Inc., New York, NY, USA, 1996.

39 M.M. Sette, P. Goethals, J. Vander Sloten and H. Van Brussel: "Tactile Sense in Minimally Invasive Surgery" in "Novel Technologies for Minimally Invasive Therapies", Lupiensis Biomedical Publications, ISBN 978-88-902880-0-5, 2007; 145-157

40 Sauerland S, Lefering R, Neugebauer EAM. Laparoscopic versus open surgery for suspected appendicitis (Cochrane Review). In: The Cochrane Library, Issue 1, 2003

41 Paganini A M, Lezoche E, Carle F, Carlei F, Favretti F, Feliciotti F, Gesuita R, Guerrieri M, Lomanto D, Nardovino M, Panti M, Ribichini P, Sarli L, Sottili M, Tamburini A, Taschieri A. A randomized, controlled, clinical study of laparoscopic vs open tension-free inguinal hernia repair. Surgical Endoscopy - Ultrasound \& Interventional Techniques. 1998. 12(7). 979-986

42 Narain P K, Moss J M, DeMaria E J. Feasibility of 23-hour hospitalization after laparoscopic fundoplication. Journal of Laparoendoscopic \& Advanced Surgical Techniques - Part A. 2000. 10(1). 5-11. 